\title{
Delayed Onset of Spinal Subdural Hematoma after Vertebroplasty for Compression Fracture: A Case Report
}

\author{
Keong Duk Lee, Hong Bo Sim, In Uk Lyo, Soon Chan Kwon, Jun Bum Park \\ Department of Neurosurgery, Ulsan University Hospital, University of Ulsan College of Medicine, Ulsan, Korea
}

Vertebroplasty (VP) is a well-known therapeutic modality used to treat pain associated with vertebral compression fractures. Major complications such as cord or root compression, epidural and subdural hematomas (SDH) and pulmonary emboli, occur in less than $1 \%$ of patients who undergo VP after compression fracture. Spinal SDH is an extremely rare complication that usually happens a few hours after the procedure. We report a case of spinal SDH that developed at two weeks after a successful VP. We also reviewed related literatures and discussed its possible pathogenesis.

Key Words: Spine $\cdot$ Subdural hematoma $\cdot$ Vertebroplasty $\cdot$ Complication

\section{INTRODUCTION}

Since Galibert et al. ${ }^{4}$, first described vertebroplasty (VP) in 1987, it has become as widely accepted technique for treating painful vertebral compression fracture, vertebral malignancy, and painful hemangiomas ${ }^{1,3,8,10,11)}$. The number of VP and kyphoplasty procedures has abruptly increased recently, due to the aging population and changing life style. There is strong evidence of pain relief and improvement in the patient's quality of life after these procedures ${ }^{1,10,13)}$. Although it is a safe procedure, adverse reaction to the bone cement, anaphylaxis, pulmonary embolism, pedicle fracture, spinal cord compression, epidural hematoma, SDH, arterial injury, vertebral body fracture, and death are the major complications that can occur after a transpedicular $\mathrm{VP}^{3,7,8,11)}$. These major complications occur in less than $1 \%$ of patients, who undergo VP after compression fracture ${ }^{3,7,8)}$. Most often the complications would develop during the operation or just after the operation. Late-developing complications such as an infection, adjacent vertebral body fractures, and recurrent fracture often appear several days to weeks following the procedure ${ }^{1,3,8)}$.

Spinal SDH is an extremely rare complication. To our knowledge, only 2 such cases have been reported ${ }^{3)}$, and they hap-

- Received: May 21, 2012 - Revised: September 21, 2012

- Accepted: September 25, 2012

Corresponding Author: Hong Bo Sim, MD, PhD

Department of Neurosurgery, Ulsan University Hospital, 290-3 Jeonha-Dong, Dong-Gu, Ulsan 682-714, Korea

Tel: +82-52-250-7139, Fax: +82-52-250-7138

Email: nssim01@gmail.com pened within 12 to 24 hours after the procedure. We experienced a case of spinal SDH occurring after VP, and treated conservatively. This case report describes a spinal SDH presenting itself two weeks after a successful transpedicular VP.

\section{CASE REPORT}

A 40-year-old female patient presented with severe low back pain after falling down the stairs. The patient was immediately admitted into another hospital. Physical examination showed tenderness on the thoraco-lumbar area. No significant neurological deficit was found. Routine biochemical and hematological tests were within normal limits. The patient underwent urgent plain radiographs, computed tomography, and magnetic resonance imaging, which showed acute compression fracture of the T11 and T12 vertebrae with mild height loss of the vertebrae (Fig. 1). Her back pain did not improved despite treatment with bed rest for 2 weeks. The patient underwent T11 and T12 transpedicular VP with continuous visualization with fluoroscopic guidance (Fig. 2). The neurological status of the patient remained intact, and she was discharged with significant improvement of her back pain on the third postoperative day.

After the VP, the patient's condition continued to improve for 2 weeks. She was readmitted to the same clinic now with acute back pain, radiating down to both legs that started suddenly when she was walking on a flat road. Her symptoms did not improve despite conservative treatment for 1 week. She was then referred to our institute. There was no recent history of trauma since the fall and no other significant medical history 


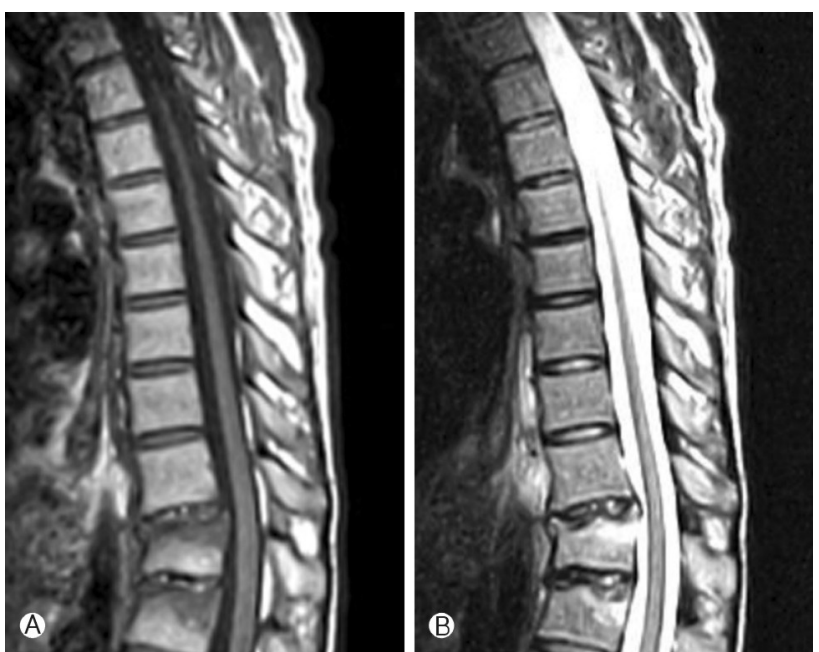

Fig. 1. T1-weighted (A), T2-weighted sagittal (B) magnetic resonance images showing acute compression fracture of the $\mathrm{T} 11$ and T12 vertebrae with mild height loss of vertebrae. There was no intradural mass lesion or cord compression.
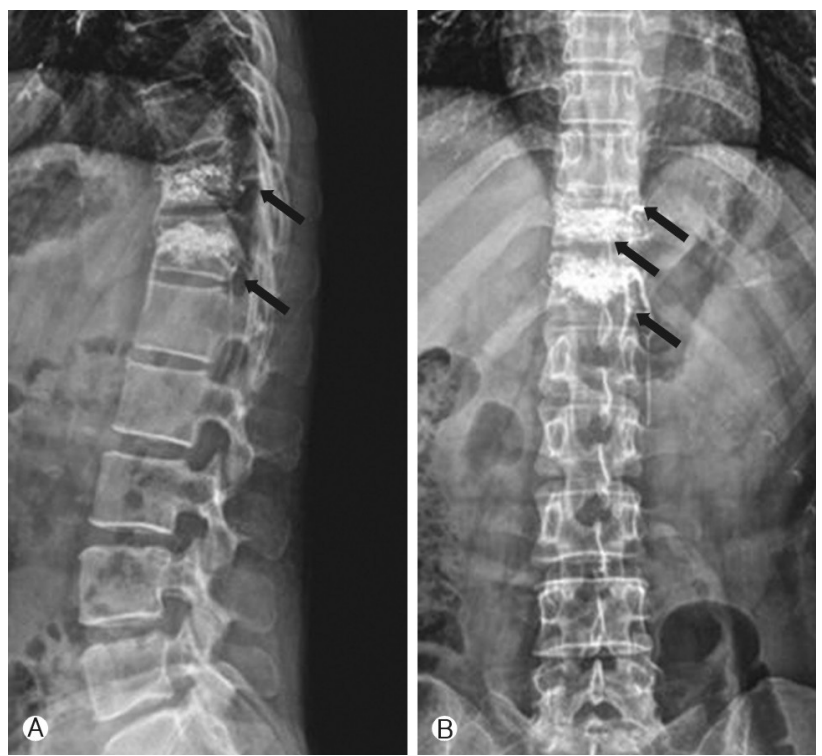

Fig. 2. Plain AP (A) and lateral films (B) after the vertebroplasty showing the bone cement filling in $\mathrm{T} 11$ and $\mathrm{T} 12$ vertebral body. Some venous leakage of bone cement into the spinal radicular veins can be seen.

except for taking oral analgesics. Neurologic examination showed intact muscle power and sensations. She had no bowel or bladder incontinence. The right straight-leg-raise test elicited pain in the leg at $40^{\circ}$ which was exacerbated by ankle dorsiflexion. $\mathrm{T} 1$ and $\mathrm{T} 2$ weighted magnetic resonance imaging was taken, which showed high signal intensity mass lesion in the intradural extramedullary space located at the low thoracic, lumbar, and sacral area (Fig. 3). Her coagulation battery
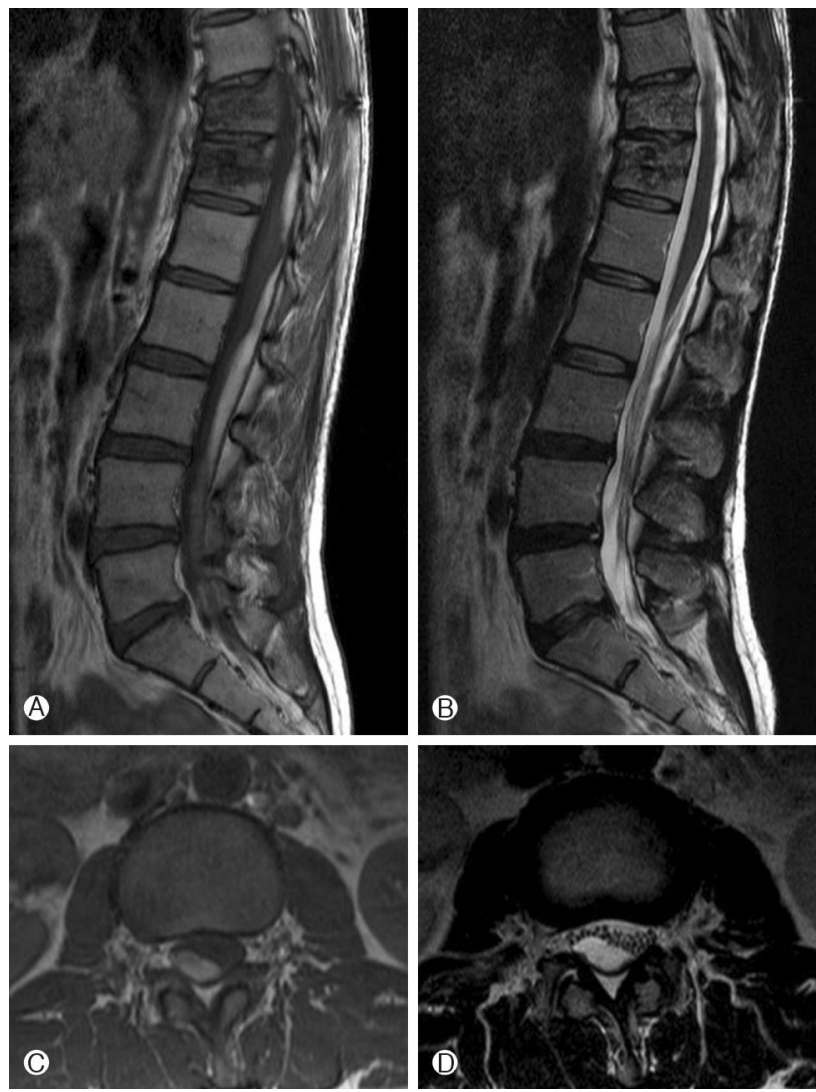

Fig. 3. A high signal intensity mass lesion is seen in the intradural extramedullary space at the low thoracic, lumbar, and sacral levels on T1-weighted (A) and T2-weighted (B) sagittal lumbar magnetic resonance image. $\mathrm{Tl}$-weighted (C) and T2-weighted (D) axial images of the L2 vertebrae level also show a high signal intensity lesion located in the dorsal aspect of the dura sac.

including platelet count, prothrombin time, and partial thromboplastin time were within normal range. Open surgery was recommended, but, she refused. Intravenous dexamethasone was given for 10 days. Her back pain and leg pain rapidly improved over 2 weeks. Magnetic resonance imaging on the $20^{\text {th }}$ hospital day revealed that the spinal SDH had almost completely resolved (Fig. 4). She was discharged without any neurologic deficit.

\section{DISCUSSION}

To our knowledge and to date, only 3 cases of spinal SDH after VP, including ours, have been reported. Cosar et al. ${ }^{3)}$, reported 2 cases that had normal neurologic examinations immediately after a successful VP and developed paraparesis developed within 12 to 24 hours later. They believed that a spinal SDH developed after puncture of the spinal dura mater and that venous blood began to slowly enter the subdural 

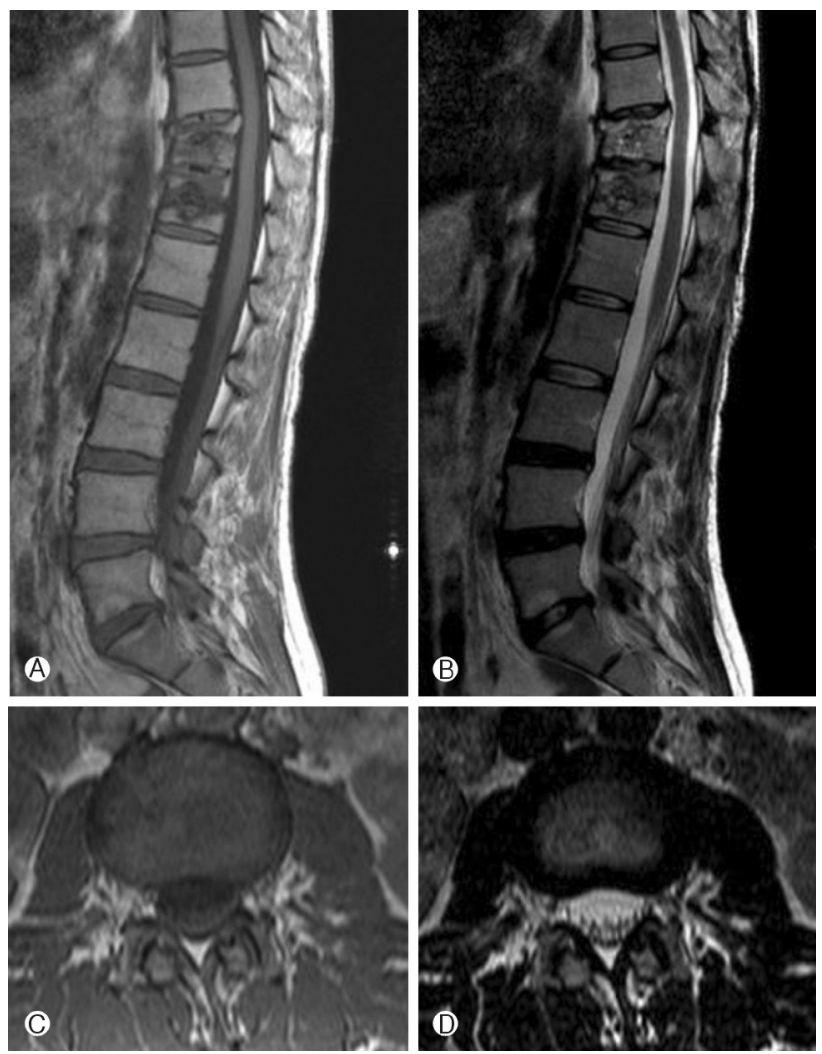

Fig. 4. The spinal subdural hematoma has almost completely resolved on the follow-up $\mathrm{T} 1$-weighted $(\mathrm{A}, \mathrm{B})$ and $\mathrm{T} 2$-weighted (C, D) sagittal resonance images done 3 weeks later.

space after this trauma. Our case is different since the symptoms in our patient developed 2 weeks after a successful VP and therefore the spinal SDH presented as a delayed onset of VP. Due to its rarity, it is evident that questions regarding the pathogenesis of delayed spinal SDH after VP remain unanswered. This may be secondary to trauma, and it is one cause of their formation.

Spinal SDH is a rare entity associated with hemorrhagic disorders, anticoagulation therapy, lumbar puncture, spinal surgery, vascular malformation, tumor, trauma, etc. ${ }^{2,5,6,9,12,15)}$. Traumatic spinal SDH is extremely rare, and less than thirty cases have been reported in the literatures. Berhouma et al. ${ }^{2}$, recently reviewed 27 previously reported traumatic spinal SDH patients including their case. They summarized the clinical presentations, specific imaging findings, pathogenesis, management and outcomes. There were several hypotheses suggested regarding the formation of a hematoma within a virtual spinal subdural space lacking bridging veins ${ }^{2)}$. A sudden and intense rise in thoracic and/or abdominal pressure may increase the pressure within the intraspinal vessels, particularly the radiculomedullary veins that cross the subdural and subarachnoid space. This, may be secondary to trauma, is one cause of their formation. Second, an abrupt cerebrospinal fluid hypotension may also lead to an indirect negative force upon the intraspinal veins, resulting in spinal SDH. Third, intracranial lesions such as intracranial $\mathrm{SDH}$, intracerebral hemorrhage, intraventricular hemorrhage, and subarachnoid hemorrhage may have a role in the pathogenesis of spinal $\mathrm{SDH}$. Forth, it may be due to the migration of blood from the intracranial subdural space into the spinal space. Last, it may directly originate from the subarachnoid space, dissecting through the arachnoid membrane and eventually break into the spinal subdural space.

Among the proposed hypotheses, we discussed its possible pathogenesis for our case. First, the theory of an increase in thoracic and/or abdominal pressure may explain what happened in ours. A sudden and intense rise in thoracic and/or abdominal pressure may increase the pressure within the intraspinal vessels, particularly the valveless radiculomedullary veins that cross the subdural and subarachnoid space ${ }^{2,9,12,14,15)}$. Mashiko et al. ${ }^{9)}$ reported a case of spinal SDH which occurred 6 days after diving that was cause by an acute increase in the intrathoracic pressure. The leakage of bone cement may increase the paraspinal veins including radiculomedullary, resulting in spinal SDH. However, this theory cannot explain ours directly. The leakage of bone cement is not so much enough to raise intraspinal venous pressure. Other possible mechanisms are the same as hypothesis of Cosar et al. ${ }^{3)}$, a spinal SDH developed after puncture of the spinal dura mater, and that venous blood began to slowly enter the subdural space after this trauma. Lastly, it may directly originate from the subarachnoid space, dissecting through the arachnoid membrane and eventually break into the spinal subdural space. Showing injuries at posterior ligaments, Spinal MRIs of our case maybe cause spinal instability and irritate dura mater, resulting delayed occurring of spinal SDH. Our suggestion has not clear evident, is only possible hypothesis. One should be vigilant that a spinal $\mathrm{SDH}$ after VP may develop, if there are many venous leakages of bone cement.

Unexpected major complications can develop after VP. To avoid major complications, proper patient selection and employing a careful and slow biplanar fluoroscopic guidance during the VP procedure are necessary. Early detection of inappropriate cement leakages can be helpful to predicting or preventing the complication.

\section{CONCLUSION}

We present a 40-year-old female, who developed a spinal SDH occurring after VP, and was treat conservatively. However, its pathogenesis is still unclear. 


\section{REFERENCES}

1. Alfonso Olmos M, Silva Gonzalez A, Duart Clemente J, Villas Tome C: Infected vertebroplasty due to uncommon bacteria solved surgically: a rare and threatening life complication of a common procedure: report of a case and a review of the literature. Spine (Phila Pa 1976) 31:E770-773, 2006

2. Berhouma M, Al Dahak N, Messerer R, Al Rammah M, Vallee B: A rare, high cervical traumatic spinal subdural hematoma. J Clin Neurosci 18:569-574, 2011

3. Cosar M, Sasani M, Oktenoglu T, Kaner T, Ercelen O, Kose KC, et al: The major complications of transpedicular vertebroplasty. J Neurosurg Spine 11:607-613, 2009

4. Galibert P, Deramond H, Rosat P, Le Gars D: [Preliminary note on the treatment of vertebral angioma by percutaneous acrylic vertebroplasty]. Neurochirurgie 33:166-168, 1987

5. Greiner-Perth R, Mohsen Allam Y, Silbermann J, Gahr R: Traumatic subdural hematoma of the thoraco-lumbar junction of spinal cord. J Spinal Disord Tech 20:239-241, 2007

6. Hung KS, Lui CC, Wang CH, Wang CJ, Howng SL: Traumatic spinal subdural hematoma with spontaneous resolution. Spine (Phila Pa 1976) 27:E534-538, 2002

7. Lee YG, Cha JH, Kim ES: Intradural bone cement leakage following percutaneous vertebroplasty. Korean J Spine 5:33-35, 2008
8. Lieberman I, Reinhardt MK: Vertebroplasty and kyphoplasty for osteolytic vertebral collapse. Clin Orthop Relat Res: S176186, 2003

9. Mashiko R, Noguchi S, Uemura K, Takada T, Matsumura A: Lumbosacral subdural hematoma. Case report. Neurol Med Chir (Tokyo) 46:258-261, 2006

10. McKiernan F, Faciszewski T, Jensen R: Quality of life following vertebroplasty. J Bone Joint Surg Am 86-A:2600-2606, 2004

11. Moreland DB, Landi MK, Grand W: Vertebroplasty: techniques to avoid complications. Spine J 1:66-71, 2001

12. Ozdemir O, Calisaneller T, Yildirim E, Caner H, Altinors N: Acute spontaneous spinal subdural hematoma in a patient with bilateral incarcerated inguinal hernia. Joint Bone Spine 75:345347, 2008

13. Park JW, Kong MH, LEE SK, Song KY, Kang DS: Improved quality of life after percutaneous vertebroplasty for osteoporotic vertebral body compression fracture in th elderly. J Korean Neurosurg Soc 34:560-564, 2003

14. Rader JP: Chronic su bdural hematoma of the spinal cord: report of a case. N Engl J Med 253:374-376, 1955

15. Sari A, Sert B, Dinc H, Kuzeyli K: Subacute spinal subdural hematoma associated with intracranial subdural hematoma. J Neuroradiol 33:67-69, 2006 